\section{Veterinary Science/Animal Model}

Feasibility of homeopathy in a flock of Zerasca sheep

M. N. Benvenuti ${ }^{1}$, F. Pisseri ${ }^{2}$, J. Goracci ${ }^{1}$, L. Giuliotti ${ }^{1}$, B. Gugliucci ${ }^{3}$, F. Macchioni ${ }^{4}$, A. Gavazza $^{3}$ and G. Guidi ${ }^{3}$

${ }^{1}$ Dipartimento Produzioni Animali, Viale delle Piagge, 2 - 56124 Pisa,

${ }^{2}$ Medico veterinario omeopata - Scuola CIMI-Koinè, ${ }^{3}$ Dipartimento

Clinica Veterinaria, Via Livornese, S. Piero a Grado and ${ }^{4}$ Dipartimento Patologia

Animale, Profilassi e Igiene degli Alimenti, Viale delle Piagge, 2 - 56124, Pisa

The control of parasitic diseases is one of the most important goals in animal health and welfare as far as environment safeguards and quality of products. Unfortunately, parasitic infections are often treated by means of chemical products, resulting in anthelmintic resistance, ecotoxicological effects and deleterious consequences on animal health. These facts underline the needs for thorough parasite monitoring and the application of integrated parasite control practices. Among these, homeopathy could play a strategic role in combating health problems; however, at present there is a lack of scientific results that might indicate validated techniques to be used on a large-scale. The aim of this study was to investigate the feasibility of homeopathy in the control of parasite burden in farm animals. The study was carried out on Zerasca breed, an Italian local sheep breed, whose farming system permits the stability and sustainability of land resources in a mountainous area in northern Tuscany. The study lasted 12 months. A homeopathic examination was performed at the beginning of the study and all useful information was collected in order to assess the farm's situation and identify the proper remedy to administer. The study involved 30 ewes randomly divided in two groups (control=C and homeopathic $=\mathrm{H}$ ). Sabadilla MK was administered twice, one after the first sampling and the other before the last one. Individual fecal and blood samples were regularly collected. Gastrointestinal strongyles fecal egg count (FEC) was performed using a modified McMaster technique. Blood count was estimated by cell counter (HeCo SEAC). Statistical analysis was performed by ANOVA. Data referring to parasites were logarithmically transformed $[y=\log (\mathrm{FEC}+25)]$ to normalize error. Correlations between FEC and hematocrit (HCT) were estimated by Pearson's correlations. FEC resulted influenced by the date of sampling confirming a fluctuation linked with the season especially in spring Homeopathy did not influence parasite burden in the whole, but a significant decrease $(P \leq 0.05)$ in egg output was observed corresponding with the first administration of the remedy. In March 2007, matching with the 'spring rise'. we could notice a lower level of $\mathrm{FEC}$ in $\mathrm{H}$ group. Data referring blood parameters did not display differences between the two groups. FEC and HCT showed an inverse correlation $(r=-0.235)$ even if mean values of HCT were included in the normal range $(28.1 \pm 3.5 \%)$. The study pointed out the advantages of a parasites monitoring and of an effective health programme limiting the use of chemical treatments with no detrimental effects on animal health.

Reduction of Neonatal Mortality in a Sheep Cohort With Homeopathic Remedies

Paolo Rossetti

Service of Veterinary Homeopathy, Center of Natural Medicine, Az USL 11, Empoli, Italy

Use of classical homeopathy in the treatment of animal diseases may be useful in the veterinary setting not only from an economical point of view, but also for the reduction of pharmacological synthetic metabolites in animal productions. In this study, we report the treatment of neonatal poisoning fo 300 Sardinian sheeps. In this sheep-breeding median neonatal mortality in the last 3 years was $8 \%$; while physiologic percentage of mortality is $2-3 \%$. Death was preceded by poor appetite, diarrhea and heavy thirst. Veterinary diagnosis was neonatal mortality due to clostridiosis. The treatment was based on the administration every 15 days of special homeopathic remedies based on Phosphorus and Zincum Phosphoricum. Mortality 6 months after the treatment was $1.5 \%$, significantly lower than physiological mortality. On the basis of these results we concluded that even if lacking a placebo controlled arm and any type of blindness is lacking, this homeopathic treatment can be considered a possible remedy for reduction of neonatal death secondary to clostridiosis in sheeps.

Anticoccidial Activity of Curcuma longa L. in Broilers

Rao Zahid Abbas, Zafar Iqbal and Muhammad Nisar Khan

Ethnoveterinary Research and Development Centre, Department of Veterinary Parasitology, University of Agriculture, Faisalabad-38040, Pakistan

Comparative efficacy of turmeric (Curcuma longa L.) crude powder and salinomycin sodium on the occurrence of coccidiosis and growth performance of broiler was evaluated. A total of 90 , day-old chicks were randomly divided into six groups. From first day onward, ration was supplemented with 1,2 and $3 \%$ turmeric powder in groups 2, 3 and 4, respectively. Group 1 received salinomycin sodium at the dose rate of $12 \mathrm{~g} / 50 \mathrm{~kg}$ feed while groups 5 and 6 were kept as infected, un-medicated and uninfected, un-medicated controls. First five groups were infected with Eimeria tenella sporulated oocysts at the dose rate of $100000 /$ chick at the age of 20 days. Body weight gain, feed consumption, feed conversion ratio were investigated throughout the experimental period, and bloody diarrhea and oocysts excretions were investigated at the first and the second week after infection. Maximum coccidiostatic effect was observed with turmeric (3\%) showing mild bloody diarrhea as compared to other infected groups receiving turmeric-containing rations. This effect was comparable with a standard coccidiostat i.e. salinomycin sodium. Similarly, the weight gain in the groups treated with salinomycin sodium $(2280 \mathrm{~g})$ and $3 \%$ turmeric $(2293 \mathrm{~g})$ were also significantly higher $(P \leq 0.05)$ than that of infected control group $(1955 \mathrm{~g})$. In the groups treated with ration supplemented with $3 \%$ turmeric powder and salinomycin sodium, the peak excretion of oocysts was delayed about 1 or 2 days relative to the control-infected group. Concentration-dependent coccidiostatic effect of turmeric suggests that further studies should be carried out to determine the possible maximum safe levels of turmeric with least toxic effects to be used as coccidiostat.

Evaluation of the Anthelmintic Activity of Caesalpinia crista (L.) Extract Against Gastrointestinal Nematodes of Sheep: In Vitro and In Vivo Studies Muhammad Arfan Zaman, Rao Zahid Abbas, Zafar Iqbal, Abdul Jabbar and Muhammad Nisar Khan

Chemotherapy Laboratory, Department of Veterinary Parasitology, University of Agriculture, Faisalabad-38040, Pakistan

The anthelmintic effect of Caesalpinia crista (L.) is described. In vitro studies were conducted on crude aqueous methanolic (CAM) extract and chloroform fraction while in vivo studies were conducted on aqueous methanolic extract and crude powder to determine possible direct anthelmintic effects of C. crista against ovine gastrointestinal nematodes. An adult motility assay and egg hatch test was used to investigate in vitro. The $\mathrm{LC}_{50}$ values of the effects of both extract and chloroform fraction were calculated. Another study was conducted in vivo to evaluate the therapeutic efficacy of the CAM extract and crude powder administered orally at dose rate of $500 \mathrm{mg}, 1,2$ and $3 \mathrm{~g} / \mathrm{kg}$ of sheep harboring naturally acquired infection of gastrointestinal nematodes. The presence of extract and its chloroform part in adult motility assay reduced the motility and survival of Haemonchus contortus. The chloroform fraction was more effective against $H$. contortus as compared to the crude extract in view of the fact that onset of anthelmintic activity from $1 \mathrm{~h}$ onward postexposure to all the concentrations of chloroform fraction in contrast to the crude extract. In the same way, the chloroform fraction was more effective in inhibiting egg hatching than crude extract. The activity of C. crista was concentration-dependent in vivo. Sheep drenched with $3 \mathrm{~g} / \mathrm{kg}$ body weight CAM extract had a mean fecal egg count (FEC) reduction of $90 \%$. The CAM extract of $C$. crista could find application in anthelmintic therapy in veterinary practices. 
A Retrospective Observational Study of Leishmania-infected Dogs: Comparison Between Homeopathic and Conventional Therapies F. Pisseri ${ }^{1}$, G. Terracciano ${ }^{2}$, A. Gavazza ${ }^{3}$, M. Bizzeti ${ }^{3}$, G. Guidi $^{3}$ and F. Mancianti ${ }^{4}$

${ }^{1}$ Scuola di Omeopatia CIMI-Koinè, Roma, ${ }^{2}$ Istituto Zooprofilattico Sperimentale Lazio e Toscana, sez. Pisa, ${ }^{3}$ Dipartimento di Clinica Veterinaria, Pisa and ${ }^{4}$ Dipartimento di Patologia Animale, Profilassi ed Igiene degli Alimenti, Pisa, Italy

Canine Leishmaniasis, a protozoan-caused disease that can be transmitted to humans, is progressively spreading in Italy, possibly due to climate changes that extends the habitat of sand fly vector. The most common treatment regimen is by $\mathrm{N}$-methylglucamine antimoniate that are unfortunately associated with side-effects such as hepatotoxicity and nephrotoxicity. Moreover, the therapy may be painful after several injections, may induce drug resistance, is expensive in the long-term, and even though it achieves resolution of clinical signs, it does not result in the complete clearing of the parasite. Clinical evolution of the disease is very variable, depending upon the type of immune response, which makes the dog susceptible or resistant to the disease. The pathogenesis is often complicated by immune-mediated phenomena that may contribute to the formation of immune complex. The characteristics of this disease make homeopathic approach valuable as either alternative or complementary therapy, since homeopathy balances adaptive reactions of the host and harmonizes its defensive systems. The aim of this study was to evaluate and compare clinical signs, clinical laboratory findings and quality of life of infected animals treated with homeopathic or conventional therapy. Sixty eligible animals divided in two homogeneous groups with respect to age, sex, breed, and clinical signs at the first examination, were included in the study. The first group (of 30) was treated with conventional therapy, and the second (of 30) with homeopathic therapy. In the latter case, therapy was tailored to each animal, due to the holistic approach that homeopathy uses to provide individual, safe and effective treatment. Inclusion criteria: IFAT titer $>1: 80$ (unambiguously positive serology): oligosymptomatic, symptomatic and asymptomatic animals present in equal number in the two groups and altered serum protein electrophoresis. Exclusion criteria: presence of concomitant infections and animals treated with alternated therapy (homeopathic and conventional). Followup evaluation was conducted by means of periodic controls of the clinical state of the animals, hematobiochemical analysis and antibody titration by IFAT. Also complications during the course of the illness was evaluated in both groups, together with therapy costs. At the first visit, the homeopathic group showed a $100 \%$ of altered a: g ratio; 12 months after, at the control, the $66.6 \%$ of subjects was in the range value. Clinical signs, in the same time, showed improved values in a percentage of $73.3 \%$. The results of the study indicate that the homeopathic therapy results in a positive effect on both clinical signs and hemotobiochemical parameters; moreover, it improves the quality of life, has no adverse effects, and its cost is low.

\section{Evaluation of the Anthelmintic Activity of Chenopodium album} Extract Against Gastrointestinal Nematodes of Sheep: In Vitro and In Vivo Studies

Muhammad Arfan Zaman, Rao Zahid Abbas, Zafar Iqbal, Abdul Jabbar and Muhammad Nisar Khan

Chemotherapy Laboratory, Department of Veterinary Parasitology, University of Agriculture, Faisalabad-38040, Pakistan

The anthelmintic effect of Chenopodium album is described. In vitro studies were conducted on crude aqueous methanolic extract (CAM) and chloroform fraction of this extract while in vivo studies were conducted on aqueous methanolic extract and crude powder to determine possible direct anthelmintic effects of $C$. album against ovine gastrointestinal nematodes. An adult motility assay and egg hatch test were used to investigate in vitro. The LC $_{50}$ values of the effects of both extract were calculated. Another study was conducted in vivo to evaluate the therapeutic efficacy of the crude aqueous methanolic extract and crude powder administered orally at dose rate of $500 \mathrm{mg}, 1,2$ and $3 \mathrm{~g} / \mathrm{kg}$ of sheep harboring naturally acquired infection of gastrointestinal nematodes. The presence of extract in adult motility assay reduced the motility and survival of Haemonchus contortus. The chloroform fraction of $C$. album was more effective against $H$. contortus as compared to the crude extracts as onset of anthelmintic activity from $1 \mathrm{~h}$ onward post-exposure to all the concentrations of chloroform fraction of $C$. album in contrast to the crude extract. In the same way, the chloroform fraction was more effective in inhibiting egg hatching than crude methanolic extract. The activity of $C$. album is concentration-dependent in vivo. Sheep drenched with $3 \mathrm{~g} / \mathrm{kg}$ body weight CAM extract had a mean fecal egg count (FEC) reduction of $68 \%$. The CAM extract of $C$. album could find application in anthelmintic therapy in veterinary practices.

Deafness and Horse Therapy: A Pilot Study on 12 Pediatric Patients.

M. P. Onofri, A. Roscio, V. Giussani and S. Demartis

Center 'Vittorio di Capua' for equestrian therapy, Milano I viale Lombardia 45 20131 Milano, Italy

The beneficial effects of horse riding on human health is known since the times of Hippocrates. The use of horse for therapeutical purpose was reintroduced in France during the years 1960-70. A Centre for Rehabilitation through the help of horse is opened and active inside the gardens of A.O. Niguarda Cà Granda, a big General Hospital in Milan, since 1981.Our Centre, named Vittorio di Capua, carried out a study to verify, if the treatment by horses could help children with severe deafness to improve their capability to communicate (orally or not), hence to be integrated inside the social contest. People, born deaf, even if trained to verbal communication, do not use this channel at the top of their capacity. They may reduce their attempt to communicate with other children of the same age and become at risk of developing psycopathological disorders, as isolation, hyperactivity and attention disorders. Six children, affected by central deafness, behavioural and emotional problems and no improve in speech therapy in act, were chosen. A control group of six children of the same age, with communication and speech problems, due to psycological problems, was also selected. Before the beginning of the treatment, a video was released to show, even with the aid of sign language, the activities that the deaf children had to carry out during the treatment. The study was carried out from February 2003 to March 2004 to promote verbal, sign, body language, to increase social behavior, to improve attention and communication. After a visit and a complete evaluation by the medical equipe,the two groups began the individual treatment on the back of the horse, once in a week. After the treatment, the children together with other peer, performed activities, as preparing food for the animals. A clinical follow-up, a serial videorecordings of the treatment, three questionnaires to parents (at the beginning, in the middle and at the end), interviews with speech therapists and teachers were performed to evaluate, if communication and language in daily life was improved. Results coming from clinical data, questionnaires, videos, interviews, showed that all subjects increased their capability of communication. The improvement of verbal communication was more evident in children without central deafness, but was significant in deaf children the progress in signs and body language and in social behaviour. Good result of the treatment was due, not only to the help of the horse, but also to the integrated work of speech therapists, horse therapists and teachers.

\section{The Application of Homeopathic Method in 3136 pigs} C. De Benedictis Via Rioli n 6400049 Velletri (Rome) Italy

One of the most serious problems in modern pig farming are diseases affecting the respiratory system. Pre-natal and neonatal losses are one of the most serious problems in pig reproduction. Viral and bacterial infections of reproductive tract take place during mating and pregnancy. Different situation is found in the domestic breeding, in which pathologies affecting pigs are due to a mismanagement. In all situations the application of homeopathic method is possible with benefit for the consumer and for animal welfare. A very good knowledge of pig behavior allows a correct use of classical homeopathic method. A survey of 3136 pigs of traditional and domestic breeding farm has been carried out. 3000 pigs of an intensive pig meat breeding farm located in the Lazio region have been treated for respiratory disorders with classic homeopathy integrated with conventional therapies in half dosage. 100 sows with reproductive pathologies have been treated with homeopathy and nosodes 
corresponding to the bacterial findings in the environment, but also homotoxicolgy and traditional therapies have been used. Thirty-six cases of domestic pigs, are bred for family use and for this reason, treated only with classic homeopathy. In the barns treated also homeopathically the consumption of powdered antibiotics in the feed, after the administration of homeopathics decrease dramatically. For the treatment of sows affected of reproduction pathologies the consumption of antibiotics decreased as the neonatal mortalilty and infertility. In the single case of domestic pigs, all pathologies have been resolved with homeopathic treatments. Homeopathy can also be successfully used in traditional intensive farming. In these circumstances, we cannot dismiss the use of feeds which include drugs during the critical stages but homeopathy is a valid support because it synergizes and limits the use of antibiotics. The use of homeopathy limits environmental pollution, decreasing the residual antibiotic in the excretion. According to new directives from the EU, sample testing the permanence of residual antibiotic molecules and parasiticidal agents in the meat have begun. The health risk to humans due to contaminated food must be checked in the stable from the animal's birth in accordance with H.A.C.C.P. Moreover saving on drugs is an advantage to the breeder. Animal welfare, ethological, behavioral aspects and ethical reflections must be considered.

\section{In vivo Anthelmintic Activity of Azadirachta indica A. Juss Seeds} Against Gastrointestinal Nematodes of Sheep

Zafar Iqbal ${ }^{1}$, Muhammad Lateef ${ }^{2}$ and Abdul Jabbar ${ }^{1}$

${ }^{1}$ Chemotherapy Laboratory, Department of Veterinary Parasitology, University of Agriculture, Faisalabad 38040 and ${ }^{2}$ Veterinary Research Institute, Lahore, Pakistan

This article describes the in vivo anthelmintic activity of seeds of Azadirachta indica to justify their use in South Asia by traditional animal healers. Seeds of $A$. indica were administered as crude powder (CP), crude aqueous extract (CAE) and crude methanol extract $(\mathrm{CME})$ at the doses of 1 and $3 \mathrm{~g} / \mathrm{kg}$ to sheep naturally infected with mixed species of gastrointestinal nematodes. Crude powder and CME exhibited a dose- $(1-3 \mathrm{~g} / \mathrm{kg})$ and a time-dependen (5-15 days post-treatment) anthelmintic effect on the animals with a maximum reduction of 29.3 and $40.2 \%$, respectively in eggs per gram (EPG) of feces. CAE did not exhibit any considerable reduction in EPG indicating the pola nature of anthelmintic constituents in the plant. Levamisole $(7.5 \mathrm{mg} / \mathrm{kg})$ a standard anthelmintic agent, exhibited $99.2 \%$ reduction in EPG. The results show that the seeds of plant possess in vivo anthelmintic activity in sheep, thus justifying its folk use in helminth infestation.

Preliminary Screening of Ethnobotanicals Used by Pastoralists for Their Anthelmintic Activity Against Trichostrongylid Nematodes of Sheep A. Jabbar ${ }^{1}$, Z. Iqbal ${ }^{1}$ and M. Lateef ${ }^{2}$

${ }^{1}$ Chemotherapy Laboratory, Ethnoveterinary Research and Development Centre, Department of Veterinary Parasitology, University of Agriculture, Faisalabad-38040 and ${ }^{2}$ Veterinary Research Institute, Lahore, Pakistan

Pastoralists all over Pakistan, mainly rely on ethnobotanicals as first aid for the treatment of common ailments of their animals. They use indigenous plant preparations either in crude form or decoction to cure the affections. The present study was conducted to evaluate the anthelmintic activity of some indigenous plants. A total of 41 plants were tested in vitro using crude aqueous extracts (CAE) and crude methanol extracts (CME) of the plants on Haemonchus contortus and out of them 10 plants were subjected to in vivo studies. Six plants showed in vitro anthelmintic activity in CAE, 13 in CME and 19 both in CAE and CME while three did not exhibit anthelmintic effects in any form. Sheep naturally infected with mixed gastrointestinal nematode species were used for in vivo studies and crude powder (CP), CAE and CME 10 plants were administered as a single dose at three different concentrations i.e., 1,2 and $3 \mathrm{~kg} / \mathrm{body}$ weight. Levamisole served as positive control for both in vitro and in vivo studies. Out of all plants/forms at different doses, a tota of 10 forms were found promising results having efficacy more than $60 \%$ and three forms i.e. CP of Trachyspermum ammi at $3 \mathrm{~g}$, CP of Butea monosperma at $3 \mathrm{~g}$ and CAE of Calotropis gigantea at $3 \mathrm{~g}$ showed $78.1,80.7$ and $88.4 \%$ efficacy, respectively. There were no observed untoward effects of any plant or the form of drug used. It was also observed that all the plants/forms showed dose-dependent response. In conclusion, the use of these plants as anthelmintic in the form of water decoction and/or as CP in 'massaulas' (physic drench/balls) by the farmers is valid in the light of results of the current study as the farmers give also $60-120 \mathrm{~g}$ of single or mixed species of plants as water decoction and/or as crude powder as an anthelmintic. Further research should be carried out on identification of active principles of plants with proven anthelmintic activity, standardization of dose and toxicity studies for drug development.

Artemisia spp.: Current State and Perspectives

L. Gori, M. Lucchesi, M. Cecchi, R. Peruzzi, R. Faccin and F. Firenzuoli Center of Natural Medicine, S. Giuseppe Hospital, Empoli, Italy

The genus Artemisia belongs to the botanical family of Compositae. It has many species, and most diffused in Europe are $A$. vulgaris, $A$. annua, A. absinthium, A. dracunculus. Artemisia vulgaris L., common in Italy, is a perennial herb. The main constituents are the essential oil containing cineol and a sesquiterpenic lactone. It is used as antispasmodic and emmenagogue, while the main practical use is for moxibustion. The powder of $A$. vulgaris is burned on the skin of the patient in particular areas of the body. Artemisia L. (qinhao, sweet wormwood) is endemic in Italy too. The active principle of $A$. annua L., artemisinin, exerts not only antimalarial activity but also profound cytotoxicity against tumor cells. Candidate genes that may contribute to the sensitivity and resistance of tumor cells to artemisinins were identified by pharmacogenomic and molecular pharmacological approaches. The identified genes are from classes with diverse biological functions: regulation of proliferation, angiogenesis, apoptosis. Artesunate triggers apoptosis both by p53-dependent and independent pathways. Cell lines overexpressing genes that confer resistance to established antitumor drugs (MDR1, MRP1, BCRP, dihydrofolate reductase, ribonucleotide reductase) were not cross-resistant to artesunate, indicating that artesunate is not involved in multidrug resistance. First encouraging experience in the clinical treatment of patients suffering from uveal melanoma calls for comprehensive clinical trials with artesunate for cancer treatment in the near future. Artemisinin isolated from the aerial parts of $A$. annua $\mathrm{L}$. is a potent antimalarial drug which has a remarkable activity against chloroquine-resistant and chloroquine-sensitive strains of Plasmodium falciparum. One major issue is the the low content $(0.01-1 \%)$ of artemisinin in the plant and is a limitation to the commercial production of the drug. Many research groups have been focusing their researches on enhancing the production of artemisinin in tissue culture or in the whole plant of $A$. annua: by biotechnological strategies including in vitro tissue culture, metabolic regulation of artemisinin biosynthesis, genetic engineering and bioreactor technology. The leaves and flowering tops of $A$. absinthium are gathered when the plant is in full bloom, and dried naturally or with artificial heat. Its active substances include, two bitter elements (absinthine and anabsinthine), thujone, thujopne, tannic and resinous substances. Its traditional use has been claimed to remedy indigestion and gastric complaints, as an antiseptic and as an antipiretic. For medicinal use, the herb is used to make a tea for helping pregnant women during pain of labour, but is not advisable due to the content of toxic substances in the essential oil. Artemisia absinthium is the main constituent of the alcoholic beverage absinthe that recently is experiencing a revival after a long prohibition. Absinthe is the cause of a syndrome called 'absinthism', which was described after chronic abuse of this spirit in the 19th century. The only consistent conclusion that can be drawn from those 19th-century studies about absinthism is that wormwood oil but not absinthe is a potent agent to cause seizures. The beverage itself is probably not epileptogenic nor that the so-called absinthism can exactly be distinguished as a distinct syndrome from chronic alcoholism. Based on the current available evidence, thujone concentrations of both pre-ban and modern absinthes may not have been able to cause detrimental health effects other than those encountered in common alcoholism. Artemisia dracunculus (Russian tarragon) is a common medicinal and culinary herb with centuries of use. Artemisia dracunculus is a close relative of the French or cooking tarragon and contains components common to many herbs that are routinely consumed without reported adverse effects. However, this plant can actually be used only for culinary and not medicinal aims because there are no clinical trials showing any reliable pharmacological activity, and the essential oil contains estragole that is an hepatotoxic and carcinogenic substance. 
Acaricidal Effect of Pakistani Plant Extracts Against Cattle TickRhipicephalus (Boophilus) microplus

Zia-ud-Din Sindhu ${ }^{1}$, Zafar Iqbal ${ }^{1}$, Nicloas N. Jonsson ${ }^{2}$, Muhammad Nisar Khan ${ }^{1}$ Abdul Jabbar ${ }^{1}$ and Rao Zahid Abbas

${ }^{1}$ Department of Veterinary Parasitology, University of Agriculture, Faisalabad38040, Pakistan and ${ }^{2}$ School of Veterinary Science, University of Queensland, Australia

Tick is a major problem for cattle producers in subtropical and tropical areas where ticks, especially Rhipicephalus (Boophilus) microplus, and the disease agents that they transmit, are a constraint to cost-effective production. The most important method of control for this tick is chemical control, which is efficient but causes damage to the environment and contamination of food. In addition, the intensive use of these materials has led to development of resistance. Thus, there is an urgent need for new and alternative compounds for tick control. The objective of this study was to evaluate the acaricidal effect of plant extracts against $R$. microplus, in search of a new acaricidal compound. Fifty-six Pakistan plants were selected and aqueous-methanolic extracts of selected plant parts were prepared and dried in rotary evaporator. In vitro trials were conducted in Australia using acaricide susceptible (N-Strain) strain. Larval immersion test (LIT) was modified (syringe method) and standardized using commercial acricides. Modified LIT (syringe method) was found more sensitive and highly repeatable compared with previous in vitro bioassays. Serial dilutions of dried plant extracts were prepared in $0.2 \%$ solution of TritonX-100. Live and dead larvae were counted on day 6 after the treatment and PoloPlus computer program was used to calculate $\mathrm{LC}_{50}$ and $\mathrm{LC}_{99}$ values in terms of percentage of plant extract. Ten most effect plants in order of their activity are Nicotiana tabacum $\left(\mathrm{LC}_{99}=0.004486 \%\right)$, Piper nigrum $\left(\mathrm{LC}_{99}=0.35534 \%\right)$, Jamal ghota ( $\left.\mathrm{LC}_{99}=0.557 \%\right)$, Curcuma longa ( $\left.\mathrm{LC}_{99}=3.379 \%\right)$, Cannabis sativa $\left(\mathrm{LC}_{99}=3.789 \%\right)$, Cedrus deodara $\left(\mathrm{LC}_{99}=6.0285 \%\right)$, Fuamaria parviflora $\left(\mathrm{LC}_{99}=9.086 \%\right)$ Acacia nilotica $\left(\mathrm{LC}_{99}=9.1794 \%\right)$ Nigella sativa $\left(\mathrm{LC}_{99}=11.939 \%\right)$ and Daphne mucronata ( $\left.\mathrm{LC}_{99}=15.743 \%\right)$. These results consolidate the belief that, plants could be good candidates in the search of new compound for tick control and management of resistance in future. 


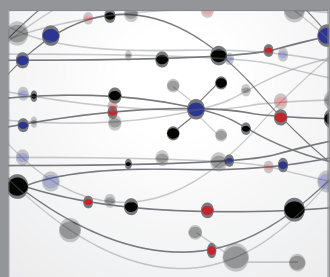

The Scientific World Journal
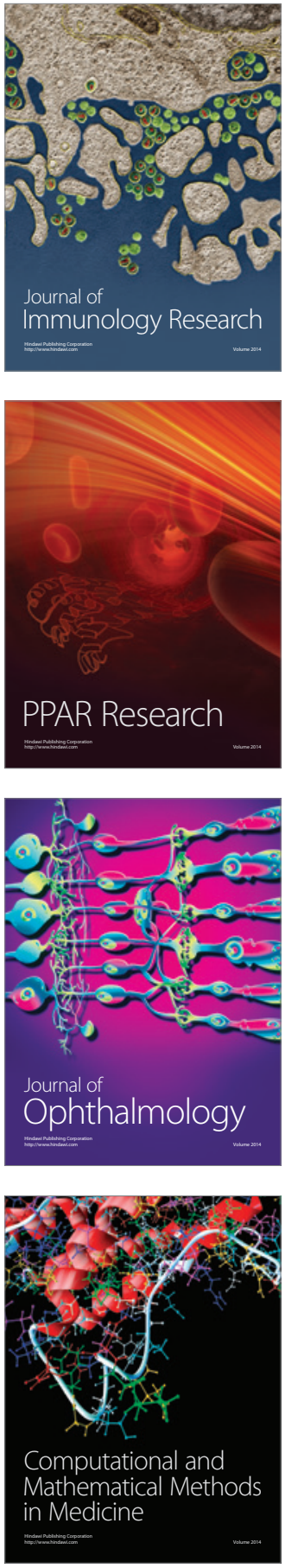

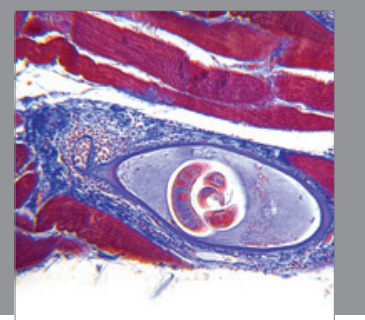

Gastroenterology

Research and Practice
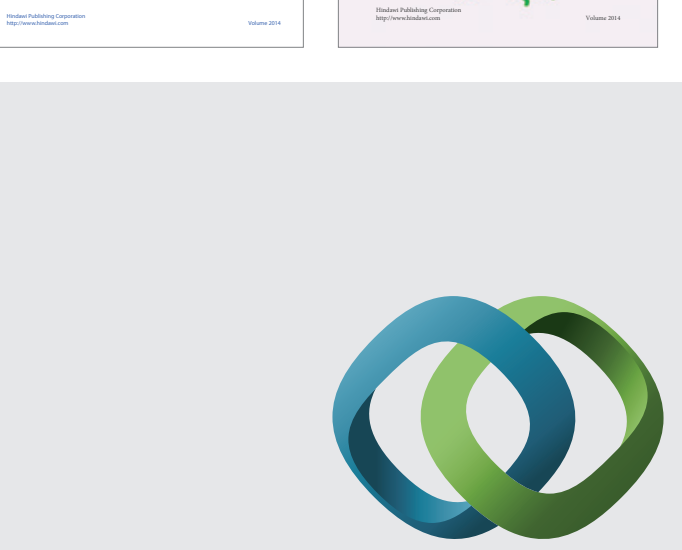

\section{Hindawi}

Submit your manuscripts at

http://www.hindawi.com
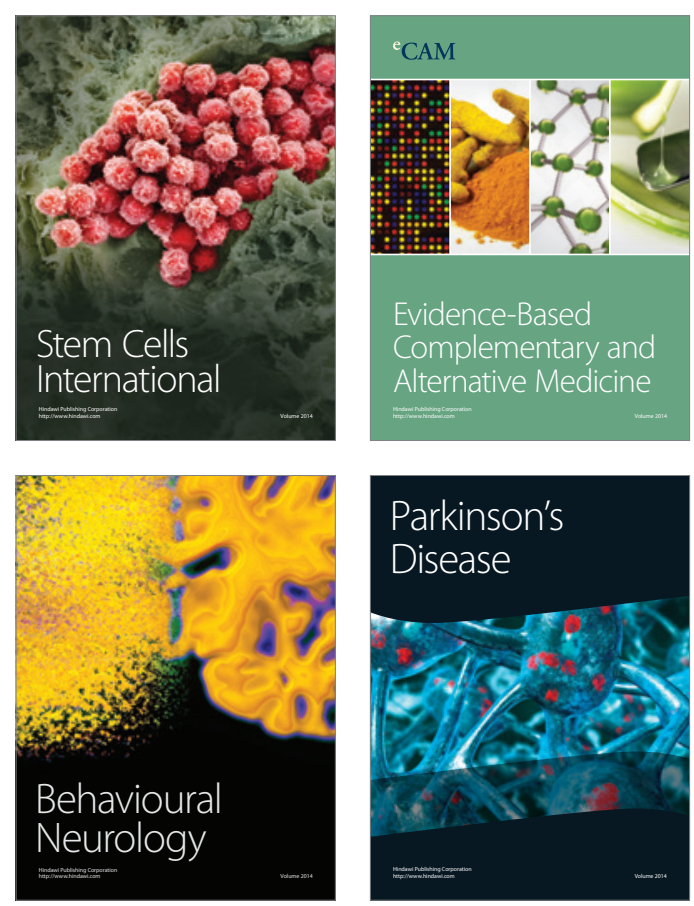

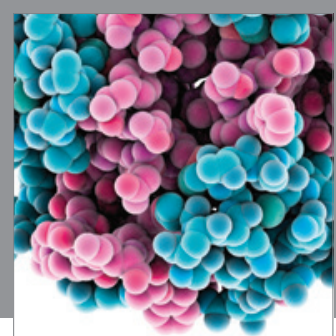

Journal of
Diabetes Research

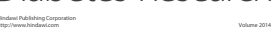

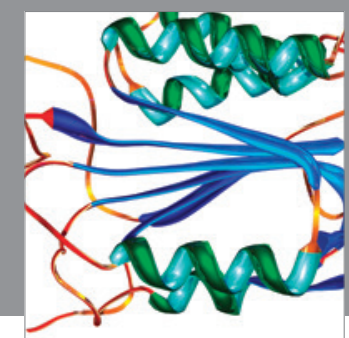

Disease Markers
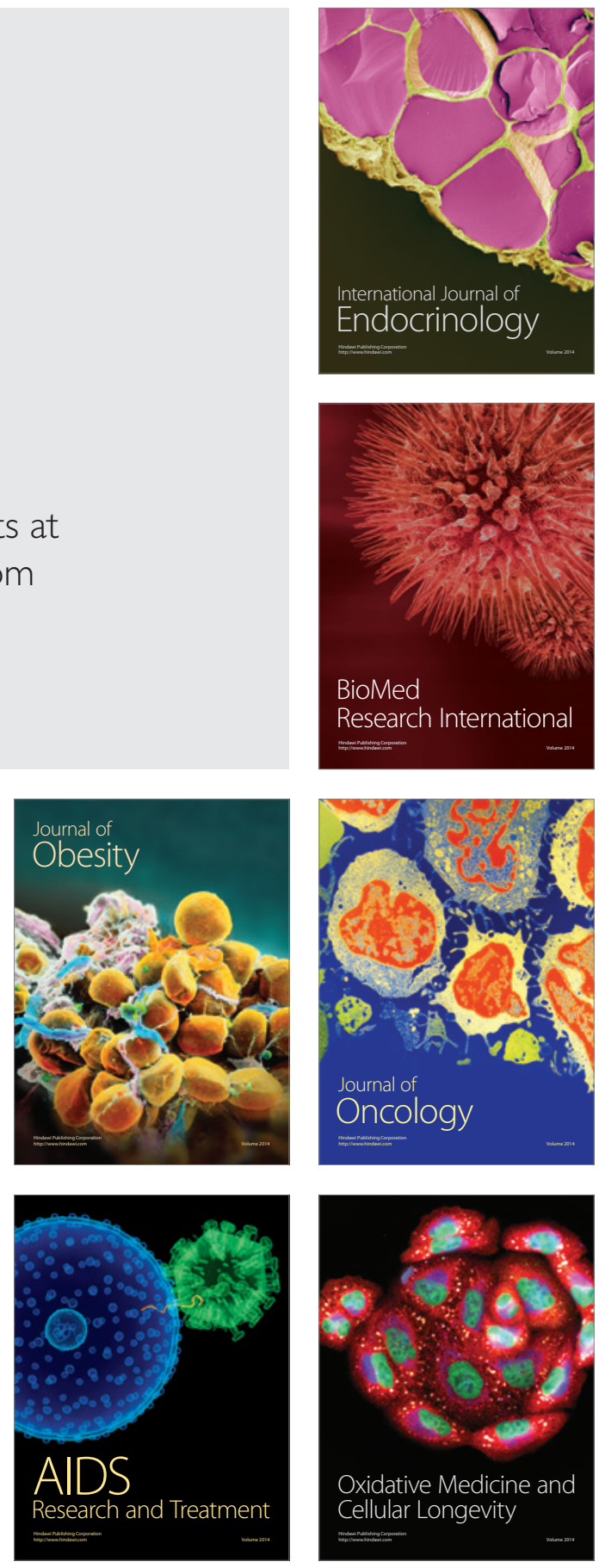